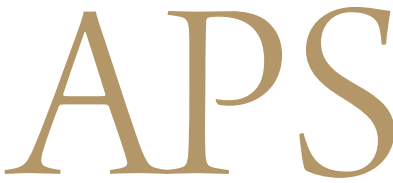

Archives of Plastic Surgery

\title{
Computer Simulation Surgery for Mandibular Reconstruction Using a Fibular Osteotomy Guide
}

\author{
Woo Shik Jeong ${ }^{1}$, Jong Woo Choi ${ }^{1}$, Seung Ho Choi ${ }^{2}$ \\ Departments of ${ }^{1}$ Plastic and Reconstructive Surgery and ${ }^{2}$ Otolaryngology, Asan Medical Center, University of Ulsan College of Medicine, \\ Seoul, Korea
}

In the present study, a fibular osteotomy guide based on a computer simulation was applied to a patient who had undergone mandibular segmental ostectomy due to oncological complications. This patient was a 68-year-old woman who presented to our department with a biopsy-proven squamous cell carcinoma on her left gingival area. This lesion had destroyed the cortical bony structure, and the patient showed attenuation of her soft tissue along the inferior alveolar nerve, indicating perineural spread of the tumor. Prior to surgery, a threedimensional computed tomography scan of the facial and fibular bones was performed. We then created a virtual computer simulation of the mandibular segmental defect through which we segmented the fibular to reconstruct the proper angulation in the original mandible. Approximately 2-cm segments were created on the basis of this simulation and applied to the virtually simulated mandibular segmental defect. Thus, we obtained a virtual model of the ideal mandibular reconstruction for this patient with a fibular free flap. We could then use this computer simulation for the subsequent surgery and minimize the bony gaps between the multiple fibular bony segments.

Keywords Surgery, plastic / Head and neck neoplasms / Mandibular reconstruction / Free tissue flaps / Computer simulation
Correspondence: Jong Woo Choi Department of Plastic and Reconstructive Surgery, Asan Medical Center, University of Ulsan College of Medicine, 88 Olympic-ro 43-gil,

Songpa-gu, Seoul 138-736, Korea

Tel: +82-2-3010-3600

Fax: +82-2-476-7471

E-mail: pschoi@amc.seoul.kr

Received: 4 Dec 2013 • Revised: 2 Mar 2014 • Accepted: 2 Mar 2014

pISSN: 2234-6163 • elSSN: 2234-6171 • http://dx.doi.org/10.5999/aps.2014.41.5.584 • Arch Plast Surg 2014;41:584-587

No potential conflict of interest relevant to this article was reported.

\section{INTRODUCTION}

The advent of new technologies has led to the replacement of many traditional treatments across different areas of clinical practice. Among these technologies, computer simulation has allowed clinicians to develop a preoperative plan for craniofacial operations and perform simulation surgeries in a virtual space that can produce a more accurate execution of the real procedure [1-4]. Traditionally, mandibular segmental defects following oncological resection have been reconstructed using fibular free flaps. For the perfect reconstruction of the mandible, an ideal mandibular contour should be designed such that the fibular bone has the contoured shape of the original mandible as well as the correct mandibular positioning for the restoration of the occlusion. For these purposes, multiple osteotomies are inevitable by using conventional procedures. However, although conventional shaping of the osteotomies is possible, a more convenient and accurate design is feasible with the use of computer simulations [5-7]. Hence, in this report, we describe the surgical treatment of a patient with a fibular osteotomy guide that was based on virtual computer simulation surgery for mandibular segmental reconstruction. 


\section{IDEA}

A 68-year-old female patient presented at our hospital with squamous cell carcinoma on her left gingival area that had been detected on a biopsy. The patient had a left gingival ulcer that produced persistent pain. Based on a previous examination with magnetic resonance (MR) T1 images, a $2.1-\mathrm{cm}$ heterogeneous enhancing soft tissue lesion was revealed with its epicenter on the left side lower gingiva. T2-weighted images further revealed

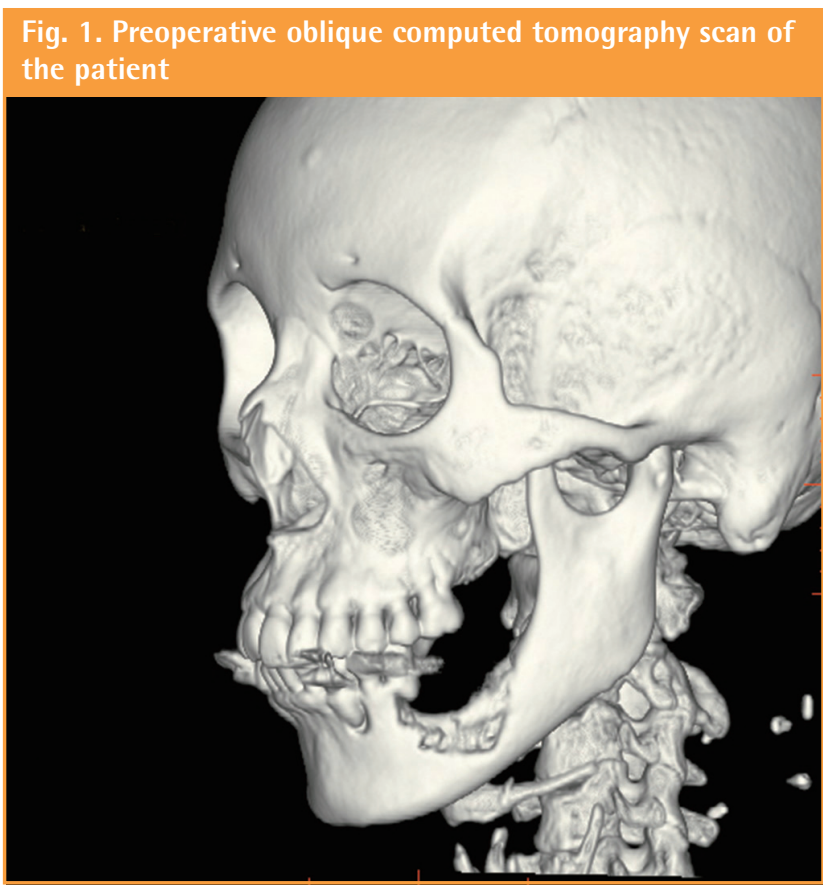

intermediate to high signal intensity for this tumor. This lesion had destroyed the cortical bony structure and caused attenuation of the soft tissue along the inferior alveolar nerve, which implied a perineural spread of the tumor. The mandibular ramus also showed signal changes and enhancement, which indicated tumor involvement with the ramal area. However, pathologic lymph nodes were not observed.

We consulted with the Head and Neck Department of our hospital regarding the treatment approach. Prior to surgery, a three-dimensional (3D) computed tomography (CT) scan of the facial and fibular bones was performed (Fig. 1). We then created a virtual computer simulation of the mandibular segmental defect through which we segmented the fibular to reconstruct the proper angulation in the original mandible. Approximately 2 -cm segments were created on the basis of this simulation and applied to the virtually simulated mandibular segmental defect (Fig. 2). Thus, we obtained a virtual model of the ideal mandibular reconstruction for this patient with a fibular free flap (Fig. 3A). For a precise reconstruction of the mandible, the correct angles between the multiple osteotomies need to be determined. We could use our computer simulation results in the subsequent surgery and minimize the bony gaps between the multiple fibular bony segments.

In order to apply our simulated results to the real surgery, a surgical guide for fibular osteotomies was produced on the basis of the virtual surgery (Fig. 3B). During the actual mandibular reconstruction with a fibular free flap for this patient, this fibular guide was used for the multiple osteotomies in the procedure

Fig. 2. Virtual computer simulation of mandibular defect

(A) Virtual computer simulation of mandibular defect and (B) application of virtually simulated mandibular segments.
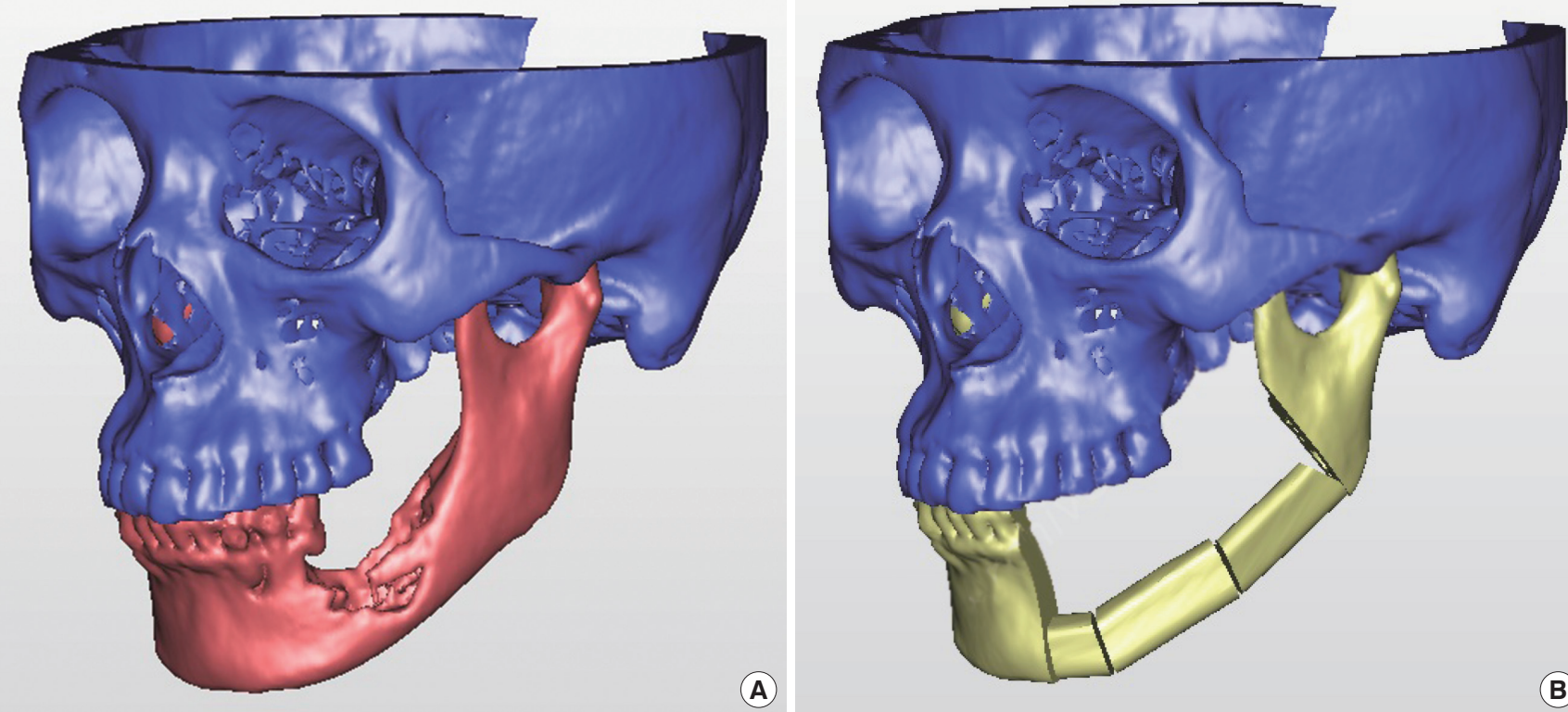
Fig. 3. RP model and fibular osteotomy guide

(A) Rapid prototype (RP) model for simulated mandibular reconstruction and (B) custom-made fibular osteotomy guide.
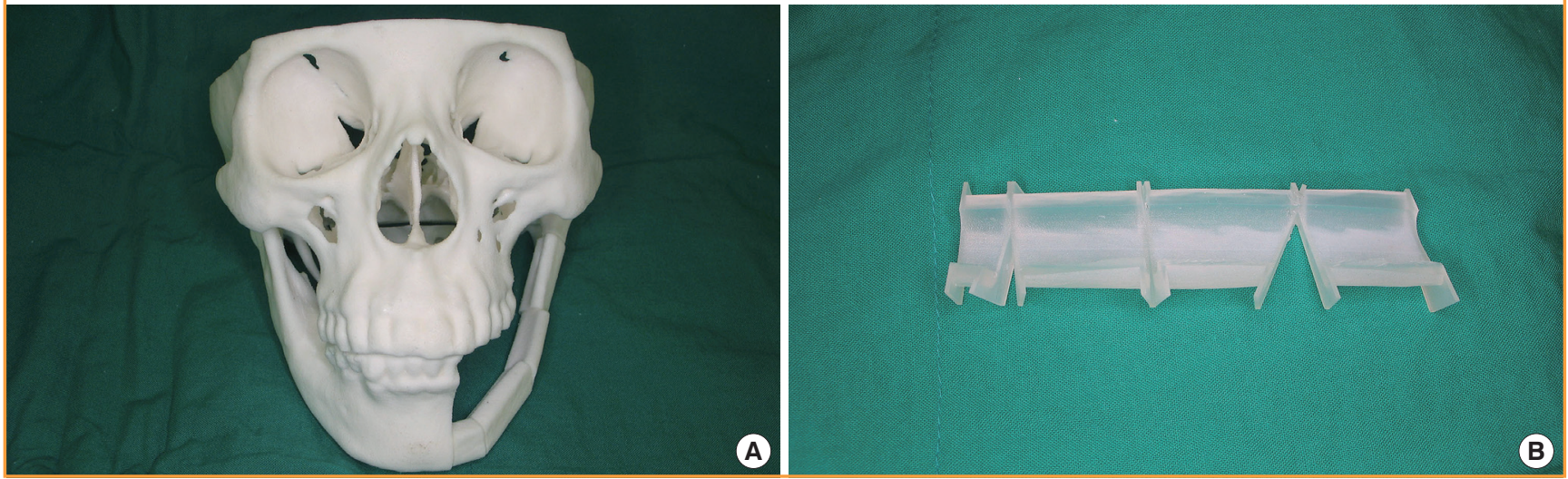

Fig. 4. Actual application of fibular osteotomy guide

(A) Actual application of a fibular osteotomy guide and (B) multiple osteotomies to a fibular osteocutaneous flap.
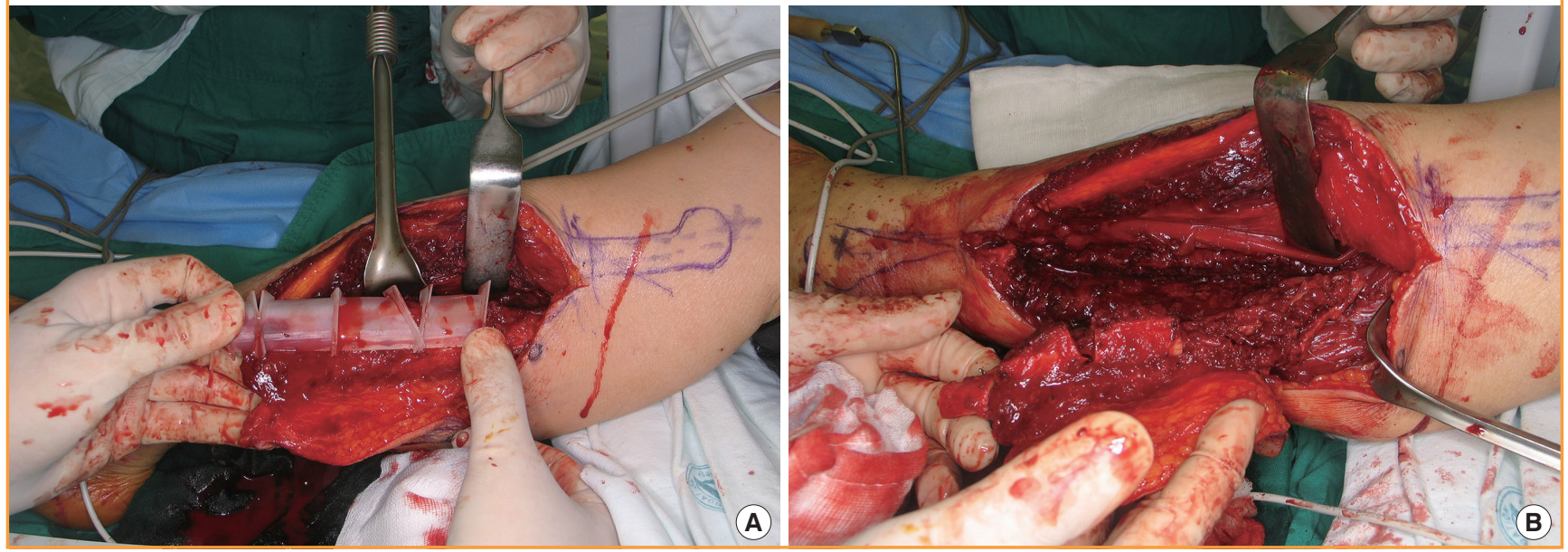

Fig. 5. Postoperative oblique computed tomography scan of the patient

The osteocutaneous fibular free flap survived and healed completely without complications after 5 months of follow-up.

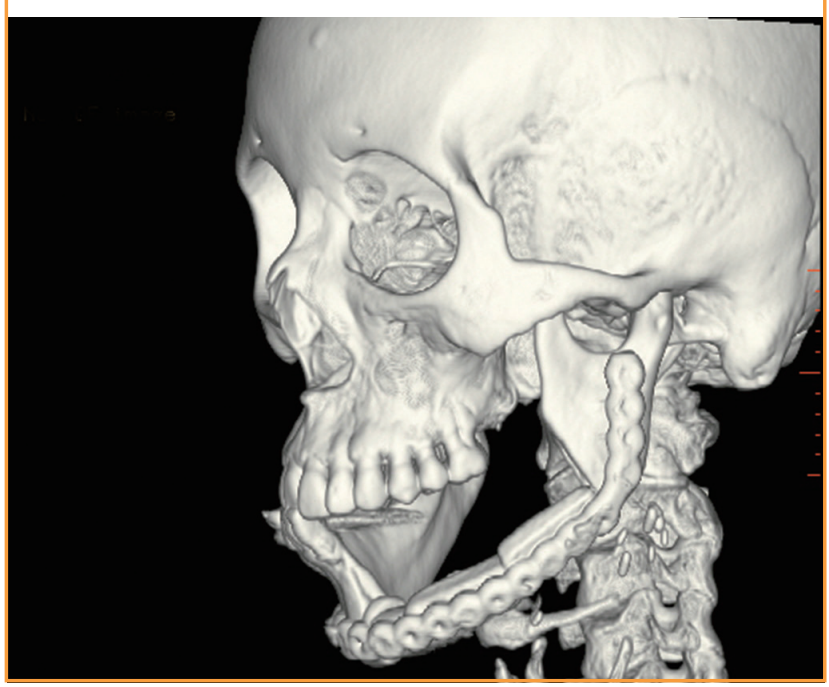

(Fig. 4). The total operation time was found to be markedly shortened compared with traditional osteotomy methods and no additional contouring or ostectomies were necessary. The osteocutaneous fibular free flap survived and healed completely without complications (Fig. 5). Our patient is scheduled to undergo dental implant surgery in the near future.

\section{DISCUSSION}

Since its introduction by Hidalgo [8] in 1989, contouring of a free fibular flap has been classically performed with a freehand approach. Although acceptable results can be obtained using this method, a learning curve on the part of the surgeon is needed for the ideal contouring of the mandible. Computer simulation surgery was introduced in the early 1990s [9]. However, despite the remarkable advancements in this technology since then, the actual application of virtual surgery has been quite limited in clinical practice. This is due to several factors that include 
the complexity involved in manipulating the computer software, the time-consuming nature of the simulation processes, high costs, and the difficulty of applying the results of the simulation surgery to real surgery. However, recent advancements in simulation technology are beginning to overcome these obstacles.

With respect to the complexity of the computer software, the manipulation process has been considerably simplified in recent years. Although some difficulties remain in this regard from a technical perspective, many of these can be overcome with the assistance of simulation experts. The problem related to the time required to conduct simulations can now be addressed with the expert assistance of computer simulation companies. These businesses are currently providing services that will greatly reduce the time that clinicians will need to spend when conducting virtual procedures. The cost of performing simulations remains an issue and can range from 1,000 to 10,000 US dollars. We have been able to reduce these costs by using a custom-made guide.

Finally, the ability to move from a simulation to an actual surgery can be addressed in two ways. One solution is to use navigational surgery that can mimic the simulation results in real surgical fields by using $3 \mathrm{D}$ positioning rods that are fixed on the skull, face, or denture. However, the cost of the navigation approach is still quite high, and precise positioning is often difficult without frequent default manipulations. The second option is the use of a surgical guide. A fibular osteotomy guide is a good example of this and can effectively serve to connect the parameters and results of simulation surgery with those of a real surgical procedure. Traditionally, this kind of surgical guide has been used in orthognathic operations as a form of surgical splint for occlusion. A surgical wafer is a tool used to connect mock surgery with an actual orthognathic procedure. Likewise, a fibular osteotomy guide has utility for this purpose. On the basis of our experiences in this current case report, we have concluded that a fibular osteotomy guide is a very useful and effective tool for performing a mandibular reconstruction with the fibular free flap. As the computer simulation technologies continue to progress rapidly, computer simulations are likely to become principal clinical and surgical tools in the near future.

This article describes our experience in surgically treating a mandibular segmental defect by using a unique computer simu- lation method developed in our clinic. The findings presented in this report indicate that the application of a fibular osteotomy guide based on simulation surgery is a feasible approach to achieving more effective, accurate, and simplified mandibular reconstructions. This is an important advance as there has been no previous report that has described the application of an osteotomy guide based on computer-simulated virtual surgery.

\section{REFERENCES}

1. Cutting C, Bookstein FL, Grayson B, et al. Three-dimensional computer-assisted design of craniofacial surgical procedures: optimization and interaction with cephalometric and CT-based models. Plast Reconstr Surg 1986;77:877-87.

2. Girod S, Keeve E, Girod B. Advances in interactive craniofacial surgery planning by 3D simulation and visualization. Int J Oral Maxillofac Surg 1995;24:120-5.

3. Mommaerts MY, Jans G, Vander Sloten J, et al. On the assets of CAD planning for craniosynostosis surgery. J Craniofac Surg 2001;12:547-54.

4. Zhang L, Sun H, Yu HB, et al. Computer-assisted orthognathic surgery combined with fibular osteomyocutaneous flap reconstruction to correct facial asymmetry and maxillary defects secondary to maxillectomy in childhood. J Craniofac Surg 2013;24:886-9.

5. Roser SM, Ramachandra S, Blair H, et al. The accuracy of virtual surgical planning in free fibula mandibular reconstruction: comparison of planned and final results. J Oral Maxillofac Surg 2010;68:2824-32.

6. Wang KH, Inman JC, Hayden RE. Modern concepts in mandibular reconstruction in oral and oropharyngeal cancer. Curr Opin Otolaryngol Head Neck Surg 2011;19:119-24.

7. Antony AK, Chen WF, Kolokythas A, et al. Use of virtual surgery and stereolithography-guided osteotomy for mandibular reconstruction with the free fibula. Plast Reconstr Surg 2011;128:1080-4.

8. Hidalgo DA. Fibula free flap: a new method of mandible reconstruction. Plast Reconstr Surg 1989;84:71-9.

9. Lo LJ, Marsh JL, Vannier MW, et al. Craniofacial computerassisted surgical planning and simulation. Clin Plast Surg 1994;21:501-16. 